\title{
Erratum to: Ultrathin flexible InGaZnO transistor for implementing multiple functions with a very small circuit footprint
}

\author{
Chaoqi Dai ${ }^{1,2,}$, Peiqin Chen ${ }^{2, \S}$, Shaocheng $\mathrm{Qi}^{2, \S}$, Yongbin $\mathrm{Hu}^{2, \S}$, Zhitang Song ${ }^{3,4}(\bowtie)$, and Mingzhi Dai ${ }^{2,3}(\bowtie)$ \\ ${ }^{1}$ College of Materials Science and Engineering, Kunming University of Science and Technology, Kunming 650093, China \\ ${ }^{2}$ Ningbo Institute of Materials Technology and Engineering, Chinese Academy of Sciences, Ningbo 315201, China \\ ${ }^{3}$ Center of Materials Science and Optoelectronics Engineering, University of Chinese Academy of Sciences, Beijing 100049, China \\ ${ }^{4}$ Shanghai Microsystem and Information Technology Institute, Chinese Academy of Sciences, Shanghai 200433, China \\ ${ }^{\S}$ Chaoqi Dai, Peiqin Chen, Shaocheng Qi, and Yongbin Hu contributed equally to this work. \\ (C) Tsinghua University Press and Springer-Verlag GmbH Germany, part of Springer Nature 2020
}

\section{Erratum to}

Nano Research 2021, 14, 232-238

https://doi.org/10.1007/s12274-020-3074-4

The article "Ultrathin flexible In GaZnO transistor for implementing multiple functions with a very small circuit footprint" written by Chaoqi Dai, Peiqin Chen, Shaocheng Qi, Yongbin Hu, Zhitang Song, and Mingzhi Dai, was erroneously originally published electronically on the publisher' internet portal (currently SpringerLink) on 30 September 2020 with caption of Fig. 1 and related context, and the Acknowledgements.

\section{Instead of}

Figure 1 (d) To implement an AND gate, the traditional design uses 3 transistors, the latest report uses 2 transistors [14], and the single transistor design uses 1 transistor.

\section{It should read}

Figure 1 (d) To implement an NAND gate, the traditional design uses 3 transistors, the latest report uses 2 transistors [14], and the single transistor design uses 1 transistor.

\section{On page 2, instead of}

As illustrated in Fig. 1(d), the conventional design of the AND logic gate has 3 transistors, whereas a recently-published design has two transistors [14]. The design here can implement the AND gate using only one transistor.

\section{It should read}

As illustrated in Fig. 1(d), the conventional design of the NAND logic gate has 3 transistors, whereas a recently-published design has two transistors [14]. The design here can implement the logic gates such as logic NAND gate or logic AND gate using only one transistor.

\section{In the Acknowledgements, instead of}

\section{Acknowledgements}

This work was supported by the National Natural Science Foundation of China (No. 61574147), Zhejiang Provincial Natural Science Foundation for Distinguished Young Scholar (No. LR17F040002), Ningbo Natural Science Foundation of China (No. 2018A610003), and Instrument Developing Project of the Chinese Academy of Sciences (No. YJKYYQ20180021). We would like to thank Editage (www.editage.com) for their English language and editing support.

\section{It should read}

Acknowledgements

This work was supported by Zhejiang Provincial Natural Science Foundation for Distinguished Young Scholar (No. LR17F040002), the National Natural Science Foundation of China (No. 61574147), Ningbo Natural Science Foundation of China (No. 2018A610003), and Instrument Developing Project of the Chinese Academy of Sciences (No. YJKYYQ20180021). We would like to thank Editage (www.editage.com) for their English language and editing support.

The online version of the original article can be found at https://doi.org/10.1007/s12274-020-3074-4 